\title{
Reflexiones acerca de la Declaración de Cancún. 2008-2018
}

\author{
Reflections on the Declaration of Cancun. 2008-2018
}

\author{
Juan Carlos de la Cruz Castillo Pineda'
}

Recibido: 1 de Agosto de 2018. Aceptado para publicación: 21 de Agosto de 2018

https://doi.org/10.35454/rncm.v1n2.045

\begin{abstract}
Resumen
La Declaración de Cancún de 2008 es un documento valiente, retador, reflexivo, pero sobre todo de unidad de las sociedades y asociaciones latinoamericanas afiliadas a la FELANPE que levanta la voz por el Derecho humano de todos los enfermos a tener una terapia nutricional óptima y oportuna brindada por personal profesional competente donde quiera que se encuentren. Gran trabajo producto de la reflexión de nutricionistas, médicos, enfermeras y químicos latinoamericanos que proponen en seis líneas de acción las alternativas para mejorar la calidad de atención nutricional de los enfermos. Ha sido objeto de análisis en diferentes universidades principalmente en Latinoamérica, y en otras latitudes ha motivado la creación de guías de práctica clínica y de servicios de Terapia Nutricional, del Consenso de Nutricionistas sobre las Funciones y Competencias del Nutriólogo Clínico, así como de la investigación de la Evaluación Nutricional en Hospitales de Latinoamérica. Aún quedan por desarrollar algunas áreas de oportunidad planteadas en la Declaración de Cancún como son: la red de investigadores latinoamericanos, la red latinoamericana de Servicios de Terapia Nutricional, las guías para la fundación de servicios de Terapia Nutricional Especializada. Lograr mayor impacto con las autoridades sanitarias y del sector privado que propicie la prevención, diagnóstico, tratamiento y seguimiento de los enfermos con compromiso del estado nutricional por carencia o por exceso, refrendar los compromisos adquiridos por los miembros de la FELANPE y generar evidencias que propicien la mejor atención de los enfermos es el reto para los próximos 10 años.
\end{abstract}

Palabras clave: declaración, derecho, terapia nutricional, salud, líneas de acción.

1 Hospital regional de Morelia, Michoacán, Instituto Mexicano del Seguro Social. Universidad michoacana de San Nicolás de Hidalgo.

Presidente FELANPE 2006-2008. México.

Correspondencia: Juan Carlos de la Cruz Castillo Pineda castillomorelia@hotmail.com

\section{Summary}

The 2008 declaration of Cancun is a brave and daring document, but it is mostly about the unification of the associations affiliated with FELANPE who raise their voice for the Human Right of all ill people to have an optimal and timely nutritional therapy, wherever they are, administered by professional and competent personnel. It is a great work, the result of a reflection by Latin American nutritionists, doctors, nurses and chemists who propose 6 lines of alternative actions to improve the quality of service for sick people in Clinical Nutrition. It has been analyzed by different universities mainly in Latin America, but also in other places, has motivated the creation of clinical practice guidelines and nutritional therapy services, besides the consensus of nutritionists on the role and competence of the clinical nutriologist and the research of Nutritional Evaluation in Latin American hospitals. There are still many areas of opportunity stated by the declaration of Cancun like the Latin-American research network, the Latin-American Nutritional Therapy Services network, the guidelines for the opening of Specialized Nutritional Therapy services and to achieve more impact on health authorities and the private sector which encourages prevention, diagnosis, treatment and follow up of sick people who have an affected nutritional state be it lacking or excess of it. The challenge for the following 10 years is to re confirm the commitment of the members of FELANPE and to generate evidence which can improve patient care.

Keywords: Declaration; Rights; Nutritional therapy; Health; Lines of action. 
Fue un gran trabajo de miembros de la FELANPE que por más de siete meses preparó este documento histórico. Vicepresidentes regionales, coordinadores de las seis mesas de trabajo generaron un anteproyecto que fue analizado y discutido por más de 75 distinguidos miembros de las sociedades de la FELANPE en una sesión extraordinaria en la que se redactaron las conclusiones que forman la parte esencial del documento de 23 páginas.

Sería difícil precisar cuándo empezó la idea o a quien se le ocurrió. Con frecuencia era tema de reuniones en cursos y congresos. Los trabajos de Jens Kondrup ${ }^{(1)}$, Stanley Dudrick, Peter Fürst, las conferencias de Lee Varela o de Sonia Echeverri, Víctor Sánchez, José Antonio Ruy Díaz, Arturo Vergara, Gustavo Kliger, Eduardo Ferraresi, Humberto Arenas y muchos otros que no alcanza la memoria ni el papel para reconocer su pasión por la atención de los enfermos y de que estos recibieran una terapia nutricional adecuada.

Existe un común denominador entre la Carta de los Derechos Humanos $^{(2)}$, la FELANPE y la Declaración de Cancún y es el número 8. La primera es de 1948, la segunda se funda en 1988, la tercera se declara en 2008. En la numerología el número 8 significa, sobre todo, comienzo, la transición entre el cielo y la tierra, el infinito, la justicia y la equidad. Estas fechas han sido fundamentales y para nosotros, la FELANPE, surge de la necesidad de llevar la buena nueva a nuestros enfermos, de que hay un grupo de profesionales en la salud que busca mejorar la calidad de la atención a través de la Nutrición Clínica.

La Declaración de Cancún nos une en un himno en torno a nuestros enfermos: "Nosotros los Presidentes de la Sociedades y Asociaciones de Nutrición Clínica y Terapia Nutricional afiliados a la Federación Latinoamericana de Terapia Nutricional, Nutrición Clínica y Metabolismo (FELANPE) ... declaramos que es nuestro deseo y compromiso comunes para garantizar que todo enfermo sea valorado nutricionalmente al ingreso a los servicios de salud de primero, segundo y tercer nivel de atención del sector público, privado o de asistencia social del mundo y en especial de Latinoamérica y que reciba una atención nutricional oportuna, completa, suficiente y de calidad, proporcionada por profesionales de la salud capacitados, que prevenga el riesgo de la desnutrición hospitalaria y reduzca el número y la severidad de las complicaciones relacionadas con el proceso de la enfermedad que llevó al paciente a hospitalizarse, además de mejorar su calidad de vida, su sobrevida y reducir los costos relacionados con la atención de la enfermedad en estos hospitales. Así también a los enfermos que por exceso y desbalance en su alimentación o por alteraciones en su metabolismo cursan con sobrepeso y obesidad dado que en la actualidad se considera una verdadera epidemia que afecta a toda la población incrementando las complicaciones médicas, aumentando los ingresos y las complicaciones hospitalarias, la estancia en el hospital y que requiere tanta atención como el desnutrido"(3).

El respeto a la institución demostrado por la confianza en los presidentes quienes en nuestro nombre declararon "deseo y compromiso..." dos términos fundamentales del ejercicio profesional. El deseo como un acto de entrega y gratuidad para la persona que lo motiva, los enfermos, y que además hace compartir la promesa de que nuestro actuar será en beneficio de los pacientes (juramento hipocrático). Este es el primer punto de reflexión de estos 10 años. Es posible que nuestras sociedades, que ahora son más, se mantengan vigentes por ese deseo y compromiso no solo con el doliente, sino también con los nuevos hermanos y discípulos que acuden a los cursos y congresos de la FELANPE. Hoy la Declaración de Cancún es un referente obligado en la mayoría de cursos de pregrado y posgrado en muchas Universidades de América Latina.

El estudio ENHOLA (2016) ${ }^{(4)}$ demostró que tanto el tamizaje como la valoración nutricional son ahora más frecuentes y que documentar en el expediente clínico aspectos nutricionales se incrementó en $22 \%$. Cabe destacar el gran trabajo que desarrolló el grupo de nutricionistas de la FELANPE que desde 2008 dictaron el primer curso para nutricionistas y que fue la base para que en 2010 presentaran un documento de las funciones y competencias del nutricionista clínico que fue una de las necesidades expresadas en las mesas de la Declaración de Cancún ${ }^{(3,5)}$. Sin embargo, no tenemos evidencia de que la Terapia Nutricional Especializada se esté utilizando con la frecuencia deseada y necesaria lo que nos reta a mejorar las estrategias de difusión y educación en el personal de la salud, pero principalmente entre los médicos. Ha faltado el desarrollo de proyectos comunes de investigación que nos permita estandarizar métodos de tamizaje y evaluación nutricional en grupos específicos, desarrollo de nuevos instrumentos y análisis de resultados de nuestros protocolos de manejo. El compartir estas experiencias nos hará ser una FELANPE más madura.

A pesar de este crecimiento, muchos de los enfermos aún no tienen la atención oportuna, completa, suficiente y de calidad. Hay avances en diferentes hospitales donde se han desarrollado equipos de Terapia 
Nutricional Especializada con protocolos que han sido reconocidos por propios y extraños, certificados por la autoridad sanitaria, pero esto no es suficiente ya que nuestro compromiso fue "que todos los enfermos..." y ahí está el reto. La malnutrición al ingreso al hospital, en la práctica, sigue sin cambios en su prevalencia y ha faltado evaluar el estado nutricional al egreso, la eficiencia de la intervención a través de un análisis crítico de resultados y sobre todo su repercusión en la evolución domiciliaria. Cada día hay más nutricionistas preparados en el campo de la nutrición clínica y son ellos quienes están generando el cambio, pero el reto no es solo en la erudición científica sino en fomentar la creación de equipos multidisciplinarios: nutricionista-enfermerafarmacéutico-médico como un gran cuarteto de cuerdas que necesitará crecer con las otras especialidades del área de la salud e incorporar a la gastronomía clínica que favorecerá el logro de metas y mejores resultados.

La obesidad y las patologías derivadas del síndrome metabólico fueron incorporadas a la FELANPE desde el Congreso de Cancún 2008 y su estudio ha ido creciendo en cada congreso, atrayendo a más profesionales que enriquecen el conocimiento, o debaten, aclaran dudas por el bien del enfermo. El estudio ENHOLA 2016 demostró que $45 \%$ de los pacientes hospitalizados tienen sobrepeso u obesidad como una comorbilidad altamente prevalente por lo que es necesario revisar en forma adecuada las modificaciones de atención a este grupo específico con el fin de disminuir las complicaciones relacionadas.

La Declaración de Cancún sigue tan vigente como hace 10 años. ¿Qué impacto ha tenido entonces en las diferentes sociedades y asociaciones miembros de la FELANPE? Sabemos que en diferentes países se ha presentado a las autoridades sanitarias, que en otros hospitales ha favorecido la apertura de nuevos servicios de Nutrición Clínica, que es revisada en diversos cursos, pero no contamos con información precisa por lo que no será fácil hacer un análisis objetivo.

La misión educativa de la FELANPE hace realidad los ideales de la Declaración con los congresos regionales, el Curso Interdisciplinario de Nutrición Clínica (C.I.N.C.) liderado por Sonia Echeverri y su excelente grupo de trabajo que ha mantenido la actualización de los materiales didácticos y que han sido exitosos en diferentes países. El Curso de Nutrición Pediátrica (C.N.P) ha sido muy solicitado, pero aún no logra la consolidación que se esperaba. Otra gran oportunidad educativa ha sido el Curso Avanzado de Nutrición Clínica donde fue posible llevar a grandes profesores latinoamericanos, europeos y americanos a las aulas de universidades y congresos. Fomentar el uso de las redes y técnicas de comunicación propios de la FELANPE facilitará la actualización de nuestros miembros en prácticamente cualquier sitio de Latinoamérica.

En las cuatro primeras mesas de trabajo se destacó la importancia de la formación de equipos multidisciplinarios en Nutrición Clínica en los hospitales para garantizar la seguridad del paciente. Será de gran utilidad crear una red de estos equipos para ayudar a los que van empezando, para comparar resultados, compartir casos, etc., además de incentivar el desarrollo de guías de práctica clínica o proyectos de investigación. La FELANPE puede ser el medio que favorezca esto a través de cursos, talleres y manuales de operación, entre otros. En esta red es posible conocer si se han desarrollado Guías de Práctica Clínica (en México se han escrito tres guías después de la Declaración de Cancún $2008^{(6-8)}$ ) y crear un repositorio de publicaciones científicas que será un gran estímulo para los autores y ayuda para los lectores. No basta organizar un exitoso congreso cada dos años sino que esta experiencia debe retroalimentar las aulas de las universidades y hospitales creando la verdadera red del conocimiento en la nutrición clínica.

En el área de investigación se propuso la realización de un censo de investigadores, así como la realización de cursos de metodología de investigación. El primero no se ha realizado aún y los cursos se han dictado en varios congresos con muy buena respuesta de los participantes. Otra de las propuestas fue la actualización del estudio de malnutrición hospitalaria publicado en 2003. En la Gestión del doctor Alfredo Matos de Panamá 2010 - 2012 fue posible realizar la investigación de la Evaluación Nutricional en Hospitales de Latino América con una participación extraordinaria de 47 hospitales y logrando encuestar a 8100 pacientes de 12 países. Este trabajo requirió una preparación, análisis y revisión del protocolo en donde participaron más de 25 miembros de la FELANPE. Un ejército de nutricionistas, médicos, enfermeras y químicos que durante seis meses fueron recolectando la información que después fue analizada por un grupo de excelentes investigadores y permitió su publicación en la revista Nutrición Hospitalaria ${ }^{(4)}$ que incluso le dedicó su comentario editorial. Esta experiencia demostró que cuando hay buena voluntad la FELANPE puede desarrollar proyectos extraordinarios y que no hay obstáculo imposible de superar. 
En la mesa de economía y nutrición se hicieron grandes propuestas de algunas de las cuales han sido comentadas previamente, sin embargo, no se han desarrollado o difundido estudios latinoamericanos que evalúen costo eficacia, costo beneficio, costo efectividad y costo utilidad de la Terapia Nutricional Especializada que ayudaran a la justificación de esta modalidad de tratamiento ante los administradores de los servicios de salud públicos y privados. Esta es una gran área de oportunidad que debe aprovecharse.

Finalmente, el trabajo que ha venido realizando la FELANPE en los últimos 10 años ha sido fructífero, pero no documentado lo suficiente y esta es una verdadera amenaza porque no se puede reinventar cada 2 años una nueva estrategia. La Declaración de Cancún es un faro, es un camino, es la convicción de un grupo de servidores de la salud por el bien del enfermo. Es un compromiso que reclama resultados, que reta a la acción eficiente de los hechos y no solo de pensamientos abstractos que con frecuencia buscan las ideas por las que no se avanza, olvidándose de que solo basta una decisión para que empiece la acción. La Declaración de Cancún sigue vigente y toca ahora que nos levantemos de nuevo para ser la voz de quien no la tiene o se la negamos, para darle seguridad de que hay profesionales competentes que llevarán la mejor opción de tratamiento nutricional que sea posible en el hospital, en la casa, y que somos personas en quien confiar. Vamos a necesitar revisar las anteriores mesas de trabajo y verificar que se logró y que no. Hagamos nuevas propuestas, seamos colaborativos y generosos que la FELANPE estará esperando tus acciones por el bien de todos.

\section{Referencias bibliográficas}

1. Kondrup J. Proper Hospital Nutrition as a Human Right. Clin Nutr. 2004;23:135-7.

2. Organización de las Naciones Unidad: Declaración Universal de los Derechos Humanos. Proclamada por la Asamblea General en su resolución 217 A (iii) de 10 de diciembre de 1948.

3. Castillo Pineda JC de la C, Figueredo Grijalva R, Dugloszewski C, Ruy Díaz Reynoso JAS, Spolidoro Noroña JV, Matos A, et al. Declaración de Cancún. Nutr Hosp. 2008;23(5):413-7.

4. Castillo Pineda JC de la C, Gómez García A, Velasco N, Díaz-Pizarro Graf JI, Matos Adámes A, Miján de la Torre A. Nutritional assessment of hospitalized patients in Latin America: association with prognostic variables. The ENHOLA study . Nutr Hosp. 2016; 33(3):655-62.

5. Canicoba M, de Baptista GA, Visconti G. Funciones y Competencias del Nutricionista Clínico. Documento de Consenso del Comité de Nutricionistas de la Federación Latinoamericana de Terapia Nutricional, Nutrición Clínica y Metabolismo. Rev Cubana Aliment Nutr. 2013;23(1):146-72.

6. Guía de Práctica Clínica Seguridad en Terapia Nutricional Especializada. Nutrición parenteral y nutrición enteral. México. Instituto Mexicano del Seguro Social, 2014. Disponible en: http://www.imss.gob.mx/profesionales/ guiasclinicas/Pages/guias.asp Consultado en: julio 15, 2018.

7. Guía de Práctica Clínica. Nutrición parenteral en el paciente adulto en estado crítico. Ciudad de México: IMSS 2017 Disponible en: http://www.cenetec.salud.gob.mx/contenidos/ gpc/catalogoMaestroGPC.html Consultado en: julio 15, 2018.

8. Guía de Práctica Clínica. Desnutrición intrahospitalaria: Tamizaje, diagnóstico y tratamiento. México. Secretaría de Salud. 2013. http://www.cenetec-difusion.com/CMGPC/ IMSS-641-13/ER.pdf Consultado en: julio 15, 2018. 